\title{
Medical Image Denoising based on Log-Gabor Wavelet Dictionary and K-SVD Algorithm
}

\author{
R.M. Farouk \\ Department of Mathematics \\ Faculty of Science, Zagazig \\ University, Zagazig 44519, \\ Egypt
}

\author{
Mohammed Elsayed \\ Department of Mathematics, \\ Faculty of Science, Zagazig \\ University, Zagazig 44519, \\ Egypt
}

\author{
Mohammed Aly \\ Department of Mathematics \\ Faculty of Science, Zagazig \\ University, Zagazig 44519, \\ Egypt
}

\begin{abstract}
Medical image denoising is the main step in medical diagnosis, which removes the noise without affecting relevant features of the image. There are many algorithms that can be used to reduce the noise such as: threshold and the sparse representation. The K-SVD is one of the most popular sparse representation algorithms, which is depend on Orthogonal Matching Pursuit (OMP) and Discrete Cosine Transform (DCT) dictionary. In this paper, an algorithm for image denoising was designed to develop K-SVD by using Regularized Orthogonal Matching Pursuit (ROMP) over log Gabor wavelet adaptive dictionary. To evaluate the performance of the proposed techniques, the results were compared with the results of DCT and Gabor wavelet dictionary. The numerical results show that the performance of our algorithm is more efficient in medical image denoising.
\end{abstract}

\section{Keywords}

Sparse representation (SR), K-SVD, log-Gabor wavelet dictionary, regularized orthogonal matching pursuit and orthogonal matching pursuit.

\section{INTRODUCTION}

In the recent years, image denoising had more attention because, it was considered as a preprocessing step in many applications such as: feature extraction, image segmentation and medical image applications [1]. In medical applications, the process of imaging acquisition can be affected by noises and artifacts. This will lead to degrading the contrast resolution of images and making medical images difficult to interpret. The denoising of medical image algorithms is needed to achieve best diagnosis without affecting relevant features of the image [1-3]. There are many types of denoising depend on different criteria such as: threshold or statistical measures. In the threshold methods, the coefficients that are lower than a certain threshold set to zero and higher than this threshold remains constant [4-6]. The threshold methods are effective in image denoising, but some of relevant features in the image lost.

Also, there are many algorithms depend on statistical measures are used to reduce the noise such as: The median filter is effective in eliminating noise, where it replaces the middle pixel in the window with the median value of its neighbors [7]. Also, maximum-likelihood filter and Bayesian denoising method is used to remove the noise models and adopt the robust parametric estimation approaches [8, 9]. However, these filters suffer from some drawbacks such as: they can remove the relevant feature from the image and also the blurring problem. To solve these drawbacks, the sparse representation (SR) was used [10]. There are a lot of advantages to use the SR such as: it will require less space on the computer and less time in computation when the value and position of entire needs to be recorded. So the SR was used in many applications such as: feature extraction [11, 12], denoising $[13,14]$, inpainting $[15,16]$, dimension reduction in image processing $[17,18]$.

There are many algorithms that have proposed to obtain the SR such as: The OMP algorithm is still one of the most effective sparse decomposition algorithms $[19,20]$. However, the OMP is a static algorithm in which, if the initial dictionary is not perfect, then the solution of OMP is not good, and to solve this problem ROMP method is used. The K-SVD algorithm is an iterative algorithm for updating the dictionary. It was used to find the best SR $[13,21]$. There are many algorithms that are developed K-SVD, for example: in [22, 23] Gabor wavelet dictionary it was used and it was found that this dictionary is better than a DCT dictionary for image denoising.

The objective of this paper is to develop K-SVD algorithm by using log-Gabor wavelet to avoid the problem in other dictionaries and also the ROMP was used to solve the drawbacks in OMP. Finally, the results of this method were compared with other methods based on different medical images.

The rest of this paper is organized as follows: Section 2 presents the sparse representation. Section 3 illustrates orthogonal matching pursuit algorithm. Section 4 introduces a description of K-SVD algorithm. In section 5, the description of log-Gabor wavelet dictionary equations is presented. Section 6 discusses regularized orthogonal matching pursuit algorithm. Finally, the numerical results of our algorithm in image denoising is discussed and compared with other methods in section 7 .

\section{SPARSE REPRESENTATION}

Sparse representation of images tends to find a set of atoms $d_{i} \in R^{n}$ that form the dictionary $D \in R^{n \times K}$ and it is defined as [13]:

$$
y_{i}=D x_{i}
$$

Where $x_{i}$ is sparse representation of $\mathrm{y}_{i}$. However, the exact solution is difficult to be found, so equation (1) can be rewritten as: $\min _{\mathrm{D}, x}\|\mathrm{y}-\mathrm{D} x\|_{2}$ s.t $\|x\|_{0}<L$

(2)

Where $L$ is a threshold that controls the sparseness of the vector $x$ and $\|.\|_{0}$ is $l_{o}$-norm that is defined as the number of non-zero entries. Another formula of the above problem is:

$\min _{\mathrm{D}, x}\|x\|_{0} \quad$ s.t $\quad\|\mathrm{y}-\mathrm{D} x\|<\varepsilon, \varepsilon \in \mathrm{R}$ 
There are many algorithms that can be used to compute the SR such as: Basis Pursuit (BP) [24] that replaces the $l_{o}$-norm with the $l_{1}$-norm and this makes the solution SR better. In other words, the Matching Pursuit (MP) algorithm selects only one atom for iteration [25]. However, the MP is time computational and to solve this, OMP is used as illustrated in next section [19].

\section{ORTHOGONAL MATCHING PURSUIT}

Orthogonal matching pursuit (OMP) is an iterative algorithm that selects at each step the atom, which is most correlated with the current residuals. At each iteration the locally optimum solution is calculated. OMP algorithm assumes that residual vector is equal to the vector that is required to be approximated (i.e. $r_{0}=y$ ). OMP algorithm can ensure that no zero components are selected. The algorithm attempts to reduce the error in representation for iteration. This is done by selecting the best atom from the dictionary that has the largest correlation with residual. The final OMP algorithm is given in algorithm 1[22].

\begin{tabular}{|l|}
\hline Algorithm 1 OMP \\
\hline Input: Dictionary $D$, stopping criterion $\varepsilon$ and $y$. \\
Output: Approximation vector $c$. \\
1. Initial $r_{0}=y, t=0$ and Index set $V_{0}=\emptyset$ \\
2. Let $v_{t}=i$ where $d_{i}$ gives the solution of max $<r_{t}, d_{k}>$ \\
3. Update the set $V_{t}$ with $v_{t}: V_{t}=V_{t-1} \cup\left\{v_{t}\right\}$ \\
4. Solve the least-squares problem $\min _{c \in C^{V_{t}} \|} \| y-$ \\
$j=1 t c(v j) d v \| 1 \mid 2$ \\
5. Calculate the new residual using $c, r_{t}=r_{t-1}-\sum_{j=1}^{t} c\left(v_{j}\right) d_{v_{j}}$ \\
6. Set $t \leftarrow t+1$ \\
7. Check stopping criterion if the criterion has not been \\
satisfied then return to Step 2 . \\
\end{tabular}

\section{K-SVD ALGORITHM}

In this section, the K-SVD algorithm is exposed for training of dictionaries. This algorithm can be flexible and also operates together with ROMP algorithm. First, dictionary $D$ was fixed and the best coefficient matrix $X$ that can be found. Coefficient matrix $X$ can be founded by using ROMP method. The second stage is conducted to look for a better dictionary. This procedure updates one column at a time, fixing all columns in $D$ other than one, $d_{k}$ and finding a new $d_{k}$ and new values for its coefficients that will reduce the MSE (mean square error). Sparse representation formulation was extended to include the entire set of observed images denoted simply by the set $Y=\left\{\left.y_{i}\right|_{i} \in[1, K], y_{i} \in R^{n}\right\}$ as [21]:

(4)

$\min _{D, X}\|Y-D X\|_{F}^{2} \quad$ s.t $\quad \forall i, \quad\left\|\quad x_{\mathrm{i}}\right\|_{0} \leq T_{0}$

The penalty term can be rewritten as:

$$
\begin{aligned}
& \|Y-D X\|_{F}^{2}=\left\|\mathrm{Y}-\sum_{j=1}^{k} d_{j} x_{T}^{j}\right\|_{F}^{2}=\| \quad\left(Y-\sum_{j \neq k} d_{j} x_{T}^{j}\right)- \\
& d_{k} x_{T}^{k}\left\|_{F}^{2}=\right\| E_{k}-d_{k} X_{T}^{k} \|_{F}^{2}
\end{aligned}
$$

The SVD finds the closest rank-1 of the matrix (in Frobenius norm) that approximates $E_{k}$, and this will effectively minimize the error as defined in (5). $\mathrm{w}_{\mathrm{k}}$ Represents band of indices pointing to $\left\{y_{j}\right\}$ that use the atom $d_{k}$, i.e., where $\mathrm{X}_{\mathrm{T}}^{\mathrm{k}}(\mathrm{i})$ is actually nonzero. Thus

$$
w_{k}=\left\{i \mid 1 \leq i \leq K, X_{T}^{k}(i) \neq 0\right\}
$$

The singular value was used to update $\mathrm{d}_{\mathrm{k}}$ and $x_{\mathrm{T}}^{\mathrm{j}}$ based on $\mathrm{E}_{\mathrm{k}}^{\mathrm{R}}$, where $\mathrm{E}_{\mathrm{k}}$ was decomposed to $\mathrm{U} \Delta \mathrm{V}^{\mathrm{T}}$. The perfect solution for $d_{k}$ was defined as the first column of $U$, and the coefficient vector $\mathrm{X}_{\mathrm{T}}^{\mathrm{k}}$ as the first column of $\mathrm{V}$ multiplied by $\Delta(1,1)$.

However, The K-SVD depends on both OMP and DCT, where OMP algorithm has some drawbacks such as: it calculated local optimum at each iteration and time computation so, to solve these problems ROMP was used. Log Gabor Wavelet was used to avoid the problems that appear from other dictionaries. K-SVD can be represented as [21]:

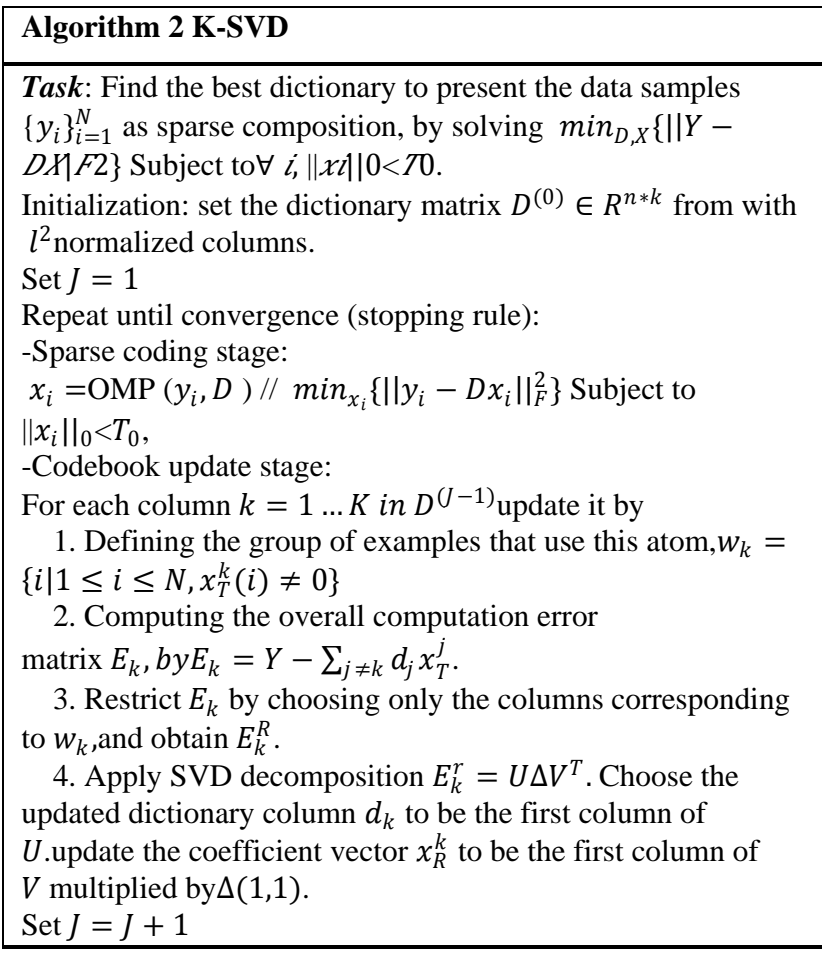

\section{LOG GABOR WAVELET DICTIONARY}

There are many types of dictionaries such as: DCT, Gabor and wavelets but exhibit major drawbacks. The log-Gabor wavelet dictionary was used to allow exactor construction and strengthen the excellent mathematical properties, for example: the higher frequency bands are covered by narrowly localized oriented filters. Also the set of filters, uniformly covers the Fourier domain, including the highest and lowest frequencies [27].

Log-Gabor wavelets get Gaussian transfer functions when viewed on the logarithmic frequency scale, however, with linear scale. This transfer perform will be asymmetric, specifically, it offers extensive tails at the higher frequency, which leads to a more efficient encoding for natural images than ordinary Gabor functions [28]. Log-Gabor wavelets don't have a DC component, and can be divided into two components: radial and angular filters and it was defined as [29]:

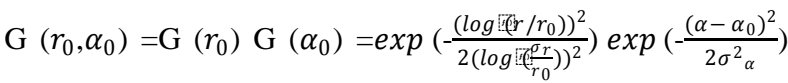


Where $r_{0}$ is the filter's center frequency, $\alpha_{0}$ is orientation angle, $\sigma_{r}$ is the radial standard deviation and $\sigma_{\alpha}$ is angular standard deviation. To get continual appearance rate filtration, the term $\sigma_{r} / r_{0}$ should be held continuously related to a lot of $r_{0}$.

\section{REGULARIZED ORTHOGONAL MATCHING PURSUIT}

ROMP algorithm uses more than one vector at each iteration [26] to form the solution and doesn't use a preset threshold ROMP algorithm. Where, in ROMP-LG the basis of Log Gabor Wavelet was used to form the atoms of dictionary. ROMP-LG was replaced by OMP. ROMP can be represented as [31]:

\begin{tabular}{|l|}
\hline Algorithm 3 ROMP \\
\hline Input: Dictionary $D$, vector $\boldsymbol{y}$ \\
Output: Approximation vector $x$ \\
1. Initialize $r_{0}=y, t=0$ and index set $V=\varnothing$ \\
2. Identify Choose a set $J$ of the $\mathrm{n}$ biggest coordinates in the \\
vector $u=D^{T} r_{t}$, (or all of its nonzero coordinates if this set is \\
smaller) \\
3. Regularize Find among all subsets $J_{0} \subset J$ the \\
maximum $\left\|\left.u\right|_{J_{0}}\right\|_{2} \cdot$ \\
Where $J_{0}$ is define $i, j \in J_{0}$ if $\left\|u_{i}\right\| \leq 2\left\|u_{j}\right\|$ and $\left.u_{i} \in u\right|_{j_{0}}$ if $k \in J_{0}$ \\
4. Update the index set by $V=V \cup J_{0}$ and residual by \\
$x=\min _{c \in R^{v}}\|y-D c\|_{2}$ \\
$r_{t+1}=y-D x$ \\
Check stopping criterion if the criterion has not been satisfied \\
then return to step 2
\end{tabular}

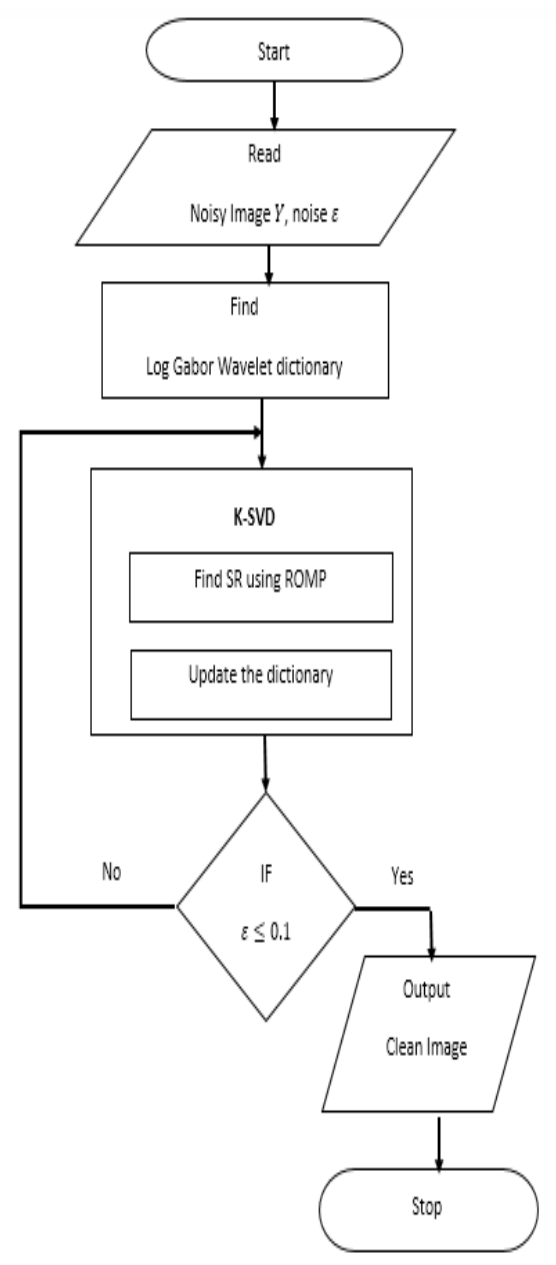

Figure 1: shows the algorithm flowchart.

This flowchart diagram can be illustrated as follows: Noisy image $Y$ and error tolerance $\varepsilon$ considered inputs to this flowchart. Formula (7) was used to calculate the log-Gabor wavelet. Column vector $d_{i, j} \in D$ is defined as the $\log$ Gabor wavelet feature vector. The Log Gabor Wavelet was used to form the atoms of dictionary. From the previous statement, vector which is defined as $d_{i, 1}, d_{i, 2}, \ldots, d_{i, n} \in D$ could be gotten $d_{i}=\left(d_{i, 1}, d_{i, 2}, \ldots, d_{i, n}\right)^{T}$ then utilized to be represented in one column of over-complete dictionary $D$. Using the above $d_{i}=\left(d_{i, 1}, d_{i, 2}, \ldots, d_{i, n}\right)^{T}$, the Over complete dictionary $D=\left\{d_{i}, i=1,2, \ldots, k\right\}$ was obtained. Then, K-SVD algorithm is used to train this type of over complete redundancy dictionary. Now the optimized over complete redundancy dictionary $D$ could be found. Testing a small sample $T$ that can be considered as a linear combination of training samples, is defined as $\mathrm{Y}=\mathrm{d}_{1,1} x_{1}+\cdots+\mathrm{d}_{\mathrm{K}, \mathrm{n}_{\mathrm{k}}} x_{\mathrm{n}}$, sparse vector $x$ is calculated through the use of ROMP method $x=\left(x_{1}, x_{2}, \ldots, x_{\mathrm{n}}\right)^{\mathrm{T}}$, where $\mathrm{n}=\mathrm{n}_{1}+\mathrm{n}_{2}+\cdots+\mathrm{n}_{\mathrm{k}}$ . Consider the noise could be written as $\mathrm{Y}=\mathrm{D} x+\varepsilon$, where $\varepsilon$ is defined as an optional error tolerance. Then, Solve problem $\quad \mathrm{Y}=\mathrm{D} x+\varepsilon$, the same as $\min _{\mathrm{D}, \mathrm{x}}\|\mathrm{y}-\mathrm{D} x\|_{2}$ s.t $\|x\|_{0}<\varepsilon, \varepsilon \in R$. If the error tolerance $\varepsilon \leq 0.1$ the image will be clean unless $\varepsilon \leq 0.1$ returns to $\mathrm{K}$ SVD method.

\section{NUMERICAL RESULTS}

To evaluate the performance of our algorithm, it was compared with other algorithms such as: KSVD based on Gabor wavelet [22], DCT [13], log-Gabor [23]. All 
experiments are performed on a PC running Windows 7 64bit and 4G RAM. The experiments are based on some benchmark MRI images dataset from [30]. The performance of the proposed algorithm was quantified across different noise levels. For each noise level, the average performance was calculated for each algorithm over 10 runs. To measure the performance of algorithms the Peak Signal to Noise Ratio (PSNR) was used and it was defined as:

$P S N R=20 \log _{10}\left(\frac{255}{M S E}\right)$

The results are illustrated in table 1 and figures 2-4. In tables 1 the K-SVD (based on ROMP) using different type of dictionaries and our algorithm are performed, in which the best results are highlighted (in bold red). From this table the $\log$ Gabor wavelet is a better one, and then log Gabor is better than others, where the original images and the image corrupted by white Gaussian noise are shown in Figures 2-4 (a) and Figures 2-4 (b) respectively. The denoising results obtained by $\log$ Gabor, our algorithm, Gabor, Gabor wavelet, DCT and wavelet are illustrated in Figures 2-4 (c)-(h) respectively.
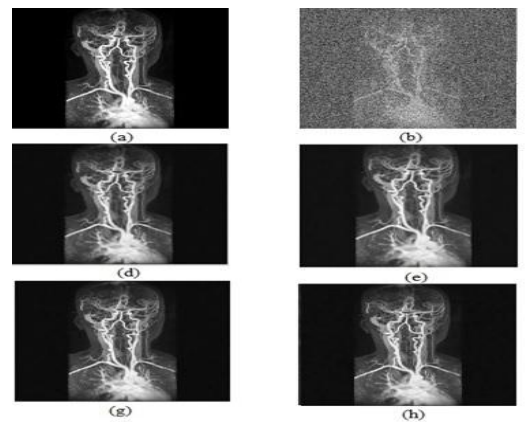

Figure 2: (a) Original image, (b) noised image, denoised image by using dictionary (c) log Gabor, (d) our algorithm, (e) Gabor, (f) Gabor wavelet, (g) DCT, (h) wavelet.
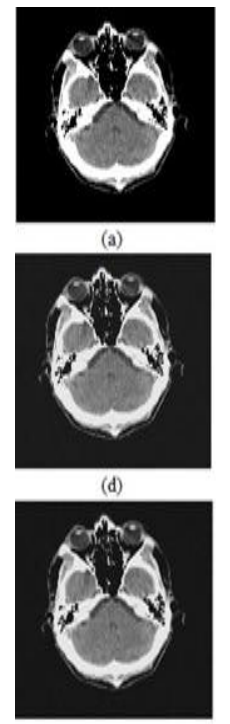

(g)

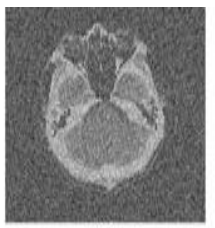

(b)
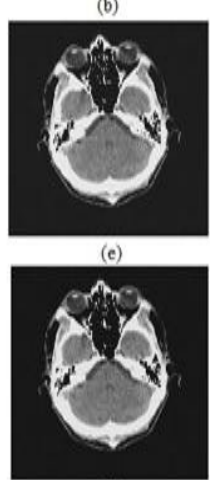

(h)
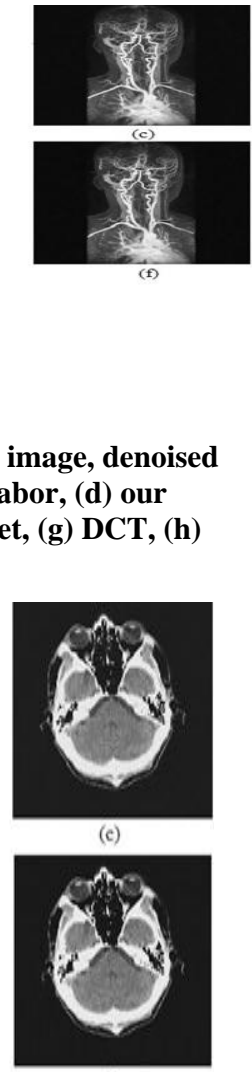

(f)
Figure 3: (a) Original image, (b) noised image, denoised image by using dictionary (c) log Gabor, (d) our algorithm, (e) Gabor, (f) Gabor wavelet, (g) DCT, (h) wavelet.

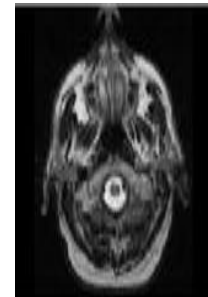

(a)

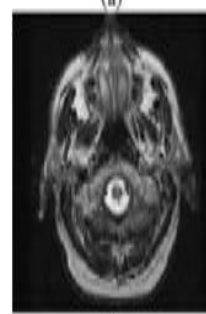

(d)

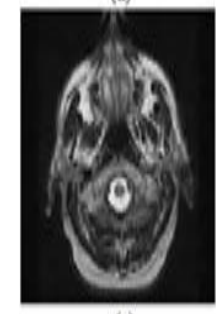

(g)

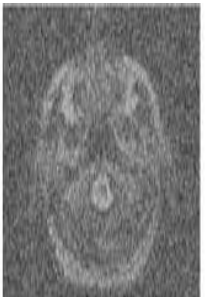

(b)

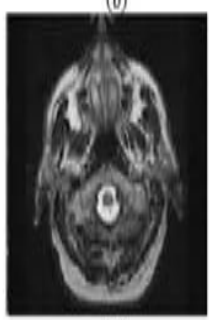

(e)

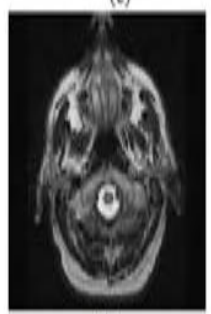

(h)

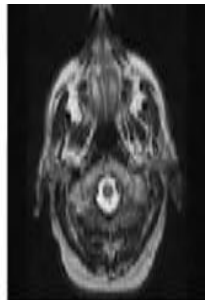

(c)

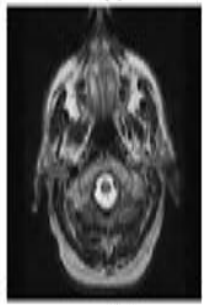

(f)
Figure 4: (a) Original image, (b) noised image, denoised image by using dictionary (c) log Gabor, (d) our algorithm, (e) Gabor, (f) Gabor wavelet, (g) DCT, (h) wavelet.

Table 1: Shows comparison between the K-SVD that developed by (log Gabor- wavelet-DCT) with different values of sigma and our algorithm.

\begin{tabular}{|c|c|c|c|c|c|c|}
\hline & $\begin{array}{l}\text { Log } \\
\text { Gabor }\end{array}$ & $\begin{array}{c}\text { our } \\
\text { algorith } \\
m\end{array}$ & Gabor & $\begin{array}{c}\text { Gabor } \\
\text { Wavelet }\end{array}$ & DCT & Wavelet \\
\hline \multirow[t]{3}{*}{$\begin{array}{l}\text { Sigma } \\
=10\end{array}$} & 37.4159 & $\begin{array}{c}37.360 \\
6\end{array}$ & 37.3855 & 37.3354 & 37.361 & $\begin{array}{c}37.340 \\
6\end{array}$ \\
\hline & 37.4381 & $\begin{array}{c}37.400 \\
7\end{array}$ & 37.3806 & 37.3656 & $\begin{array}{c}37.412 \\
1\end{array}$ & $\begin{array}{c}37.314 \\
5\end{array}$ \\
\hline & 33.3333 & $\begin{array}{c}33.340 \\
5\end{array}$ & 33.262 & 33.2786 & $\begin{array}{c}33.493 \\
3\end{array}$ & $\begin{array}{c}33.237 \\
8\end{array}$ \\
\hline \multirow[t]{3}{*}{$\begin{array}{l}\text { Sigma } \\
=20\end{array}$} & 33.6904 & 33.772 & 33.5372 & 33.646 & 33.533 & $\begin{array}{c}33.605 \\
4\end{array}$ \\
\hline & 33.6049 & $\begin{array}{c}33.720 \\
2\end{array}$ & 33.6738 & 33.5916 & $\begin{array}{c}33.637 \\
2\end{array}$ & $\begin{array}{c}33.677 \\
4\end{array}$ \\
\hline & 29.7943 & $\begin{array}{c}29.709 \\
5\end{array}$ & 29.5993 & 29.6772 & $\begin{array}{c}29.706 \\
2\end{array}$ & $\begin{array}{c}29.674 \\
7\end{array}$ \\
\hline \multirow[t]{3}{*}{$\begin{array}{l}\text { Sigma } \\
=\mathbf{3 0}\end{array}$} & 31.4444 & $\begin{array}{c}31.491 \\
7\end{array}$ & 31.4095 & 31.3366 & $\begin{array}{c}31.359 \\
8\end{array}$ & 31.476 \\
\hline & 31.4269 & $\begin{array}{c}31.543 \\
7\end{array}$ & 31.4331 & 31.3441 & $\begin{array}{c}31.334 \\
6\end{array}$ & $\begin{array}{c}31.454 \\
5\end{array}$ \\
\hline & 27.9112 & $\begin{array}{c}27.841 \\
2\end{array}$ & 27.7845 & 27.7573 & $\begin{array}{c}27.810 \\
1\end{array}$ & $\begin{array}{c}27.839 \\
8\end{array}$ \\
\hline \multirow[t]{3}{*}{$\begin{array}{l}\text { Sigma } \\
=40\end{array}$} & 29.9162 & $\begin{array}{c}29.883 \\
1\end{array}$ & 29.8153 & 29.7989 & $\begin{array}{c}29.745 \\
3\end{array}$ & $\begin{array}{c}29.843 \\
3\end{array}$ \\
\hline & 29.8327 & $\begin{array}{c}29.924 \\
2\end{array}$ & 29.8011 & 29.7985 & $\begin{array}{c}29.858 \\
2\end{array}$ & $\begin{array}{c}29.846 \\
4\end{array}$ \\
\hline & 26.6486 & $\begin{array}{c}26.605 \\
8\end{array}$ & 26.2738 & 26.4632 & $\begin{array}{c}26.458 \\
1\end{array}$ & $\begin{array}{c}26.329 \\
9\end{array}$ \\
\hline \multirow[t]{3}{*}{$\begin{array}{l}\text { Sigma } \\
=50\end{array}$} & 28.6574 & $\begin{array}{c}28.578 \\
9 \\
\end{array}$ & 28.4951 & 28.5258 & $\begin{array}{c}28.494 \\
3 \\
\end{array}$ & $\begin{array}{c}28.417 \\
4\end{array}$ \\
\hline & 28.5492 & $\begin{array}{c}28.565 \\
2\end{array}$ & 28.5339 & 28.5576 & 28.511 & $\begin{array}{c}28.501 \\
8\end{array}$ \\
\hline & 25.4815 & $\begin{array}{c}25.464 \\
2\end{array}$ & 25.2901 & 25.3413 & $\begin{array}{c}25.302 \\
2\end{array}$ & $\begin{array}{c}25.368 \\
8\end{array}$ \\
\hline
\end{tabular}




\begin{tabular}{|l|c|c|c|c|c|c|}
\hline $\begin{array}{l}\text { Sigma } \\
=\mathbf{6 0}\end{array}$ & $\mathbf{2 7 . 5 6}$ & $\begin{array}{c}27.512 \\
3\end{array}$ & 27.4365 & 27.3981 & $\begin{array}{c}27.551 \\
7\end{array}$ & $\begin{array}{c}27.394 \\
8\end{array}$ \\
\cline { 2 - 7 } & 27.4606 & $\begin{array}{c}\mathbf{2 7 . 5 2 7} \\
\mathbf{2}\end{array}$ & 27.4303 & 27.4551 & $\begin{array}{c}27.438 \\
8\end{array}$ & $\begin{array}{c}27.352 \\
4\end{array}$ \\
\cline { 2 - 7 } & 24.5113 & $\begin{array}{c}24.360 \\
3\end{array}$ & 24.3883 & 24.1102 & $\begin{array}{c}\mathbf{2 4 . 5 4 4} \\
\mathbf{2}\end{array}$ & $\begin{array}{c}24.360 \\
5\end{array}$ \\
\hline $\begin{array}{l}\text { Sigma } \\
=\mathbf{7 0}\end{array}$ & 26.4708 & $\begin{array}{c}26.549 \\
4\end{array}$ & 26.5134 & 26.5107 & $\mathbf{2 6 . 6 7 7}$ & $\begin{array}{c}26.496 \\
1\end{array}$ \\
\cline { 2 - 7 } & $\mathbf{2 6 . 6 6 7 3}$ & $\begin{array}{c}26.595 \\
5\end{array}$ & 26.5775 & 26.329 & 26.576 & $\begin{array}{c}26.463 \\
1\end{array}$ \\
\cline { 2 - 7 } & 23.6017 & $\begin{array}{c}\mathbf{2 3 . 8 4 5} \\
\mathbf{8}\end{array}$ & 23.5327 & 23.7779 & $\begin{array}{c}23.732 \\
8\end{array}$ & $\begin{array}{c}23.521 \\
9\end{array}$ \\
\hline \multirow{3}{*}{$\begin{array}{l}\text { Sigma } \\
=\mathbf{8 0}\end{array}$} & 25.7499 & $\begin{array}{c}\mathbf{2 5 . 8 7 7} \\
\mathbf{2}\end{array}$ & 25.7586 & 25.6786 & $\begin{array}{c}25.875 \\
4\end{array}$ & $\begin{array}{c}25.677 \\
6\end{array}$ \\
\cline { 2 - 7 } & 25.5956 & $\begin{array}{c}25.785 \\
2\end{array}$ & 25.6575 & 25.7074 & $\begin{array}{c}25.817 \\
\mathbf{6}\end{array}$ & $\begin{array}{c}25.707 \\
2\end{array}$ \\
\cline { 2 - 7 } & 23.1469 & $\begin{array}{c}22.912 \\
1\end{array}$ & 23.0781 & 22.9121 & $\begin{array}{c}\mathbf{2 2 . 9 5 7} \\
\mathbf{9}\end{array}$ & $\begin{array}{c}23.094 \\
1\end{array}$ \\
\hline \multirow{4}{*}{$\begin{array}{l}\text { Sigma } \\
=\mathbf{9 0}\end{array}$} & $\mathbf{2 5 . 0 9 6}$ & $\begin{array}{c}25.073 \\
6\end{array}$ & 25.0778 & 25.0834 & $\begin{array}{c}25.071 \\
3\end{array}$ & $\begin{array}{c}25.089 \\
1\end{array}$ \\
\cline { 2 - 7 } & 25.0171 & $\begin{array}{c}\mathbf{2 5 . 6 6 9} \\
\mathbf{2}\end{array}$ & 25.0211 & 24.9827 & $\begin{array}{c}25.202 \\
4\end{array}$ & $\begin{array}{c}25.014 \\
9\end{array}$ \\
\cline { 2 - 7 } & 22.4846 & $\begin{array}{c}\mathbf{2 2 . 6 8 5} \\
\mathbf{6}\end{array}$ & 22.4142 & 22.465 & $\begin{array}{c}22.491 \\
7\end{array}$ & $\begin{array}{c}22.402 \\
3\end{array}$ \\
\hline
\end{tabular}

\section{CONCLUSION}

In this paper, image denoising has been discussed using log Gabor wavelet dictionary to remove noise from medical images. Some methods based on sparse and redundant representations of images over learned dictionaries are proposed. PSNR was used to measure the performance of the different techniques. The numerical results in table 1 and figures 2-4 show that, $\log$ Gabor wavelet and ROMP method are able to outperform some of these methods in all results, taking into account the average and the best solutions found. The comparison results demonstrate that log Gabor wavelet dictionary and ROMP method provides the best results.

\section{ACKNOWLEDGMENTS}

Our thanks to the experts who have contributed towards development of the template.

\section{REFERENCES}

[1] El-Henawy, I., El-Areef, T., and Karawia A.2003 On wavelets applications in medical image denoising. Machine Graphics \& Vision International Journal, vol. 12 , no. 3 , pp. $393-404$

[2] Prudhvi, N., Raj, V., and Venkateswarlu, T. 2012 Ultrasound medical image denoising using hybrid bilateral filtering. International Journal of Computer Applications, vol. 56, no.14, pp. 44-51.

[3] Satheesh, S., and Prasad, D.2011. Medical imagedenoising using adaptive threshold based on contourlet transform. Advanced Computing International Journal (Acij), vol. 2, no. 2.

[4] Hui, T., Zengli, L., Lin, C., and Zaiyu, C.2013 Wavelet image denoising based on the new threshold function. Proceedings of the 2nd International Conference on Computer Science and Electronics Engineering (ICCSEE).

[5] Huang, L., Wang, H., and Zhu, B. 2008 Adaptive thresholds algorithm of image denoising based on nonsubsampled contourlet transform. Computer Science and Software Engineering, vol. 6, pp.209-212.
[6] Chuia, M., Fengb, Y., Wanga ,W., Lic, Z., and Xua, X.2012 Image denoising method with adaptive bayes threshold in non-sub sampled contourlet domain. AASRI Procedia, vol. 1, pp. 512-518.

[7] Shrestha, S.2014 Image denosing using new adaptive based median filter. Signal \& Image Processing International Journal (SIPIJ), vol. 5, no. 4, pp. 1-13

[8] Shankar.2006 Speckle reduction in ultrasonic images through a maximum likelihood based adaptive filter. Physics in Medicine and Biology, vol. 51, no. 21, pp. 5591-5602.

[9] Rajan, J., Jeurissen, B., Verhoye, M., Audekerke, J., and Sijbers, J.2011 Maximum likelihood estimation-based denoising of magnetic resonance images using restricted local neighborhoods. Physics in Medicine and Biology, vol. 56, no. 16 , pp. 5221-5234.

[10] Guo, Y., Chai, H., and Wang, Y.2015 A global approach for medical image denoising via sparse representation. International Journal of Bioscience, vol. 5, no. 1, pp. 2635.

[11] Wang, J., Gao, X., and Guo, Z.2013 Feature extraction based on sparse representation with application to epileptic EEG classification. International Journal Imaging System Technology, vol. 23, pp. 104-113.

[12] Kang, L., Hsu, C., Chen, H., Lu, C., Lin, C., and Pei, S.2011 Feature-based sparse representation for image similarity assessment. IEEE Trans, vol. 13, no. 5.

[13] Elad, M., and Aharon, M.2006 Imagdenoising via sparse and redundant representations over learned dictionaries. IEEE Trans Image Process, vol. 15, no. 12, pp. 37363745 .

[14] Valiollahzadeh, S., Firouzi, H., Babaie-Zadeh, M., and Jutten C.2009 Image denoising using sparse representations. Springer, vol. 5441, pp. 557-564.

[15] Ramya, C., Subha Rani, S., and Kayalvizhi, G.2014 Inpainting Based on Fast Inpainting and Sparse Representation Method. Advanced Materials Research, pp. 1350-1356.

[16] Shen, B., Hu, W., Zhang, Y., and Zhang, Y.2009 Image inpainting via sparse representation. IEEE International Conference acoustics, Speech and Signal Processing, (ICASSP), pp. 697-700.

[17] Xu, J., Yang, G., Yafeng, Y., Man, H., and He, H.2014 Sparse representation based classification with structure preserving dimension reduction. Cognitive Computation, vol. 6, no. 3, pp. 608-621.

[18] Zhang, D., Yang, M., Feng, Z., and Zhang, D.2010 On the dimensionality reduction for sparse representation based Face recognition. Pattern Recognition (ICPR), International Conference, pp. 1237-1240.

[19] Yin, Hooping.2015 Fusion algorithm of optical images and sar with svt and sparse representation. International journal on smart sensing and intelligent, vol. 8, no. 2, pp. 1123-1141.

[20] Tony, Cai., and Wang, L.2011 Orthogonal matching pursuit for sparse signal recovery with noise. IEEE Transactions on Information Theory, vol. 57, no. 7, pp. 4680- 4688 
[21] Aharon, M., Elad, M., and Bruckstein A.2006 K-SVD: An algorithm for designing over complete dictionaries for sparse representation. IEEE Trans, Signal Process, vol. 54, no. 11, pp. 4311-4322.

[22] Khedr, W.2012 Image denoising using K-SVD algorithm based on Gabor wavelet dictionary. International Journal of Computer Applications, vol. 59, no. 2, pp. 30- 33.

[23] Wang, B., Ding, D., Yang, J., and Kong, B.2014 An optimization sparse representation algorithm based on Log Gabor. International Journal of Image Processing, Image Processing and Pattern Recognition, vol. 7, no. 4, pp. 221-230.

[24] Chen, S. S., Donoho, D. L., and Saunders, M. A.1988 Atomic decomposition by basis pursuit. SIAM J. Sci. Computer, vol. 20, no. 1, pp. 33-61.

[25] Mallat, S., and Zhang, Z.1993 Matching pursuit in a time-frequency dictionary. IEEE Trans, vol. 41, no. 12, pp. 3397-3415.

[26] Azad, H., Sheikhi, A., and Masnadi-Shirazi, M. A.2013 Sparse signal reconstruction from compressed sensing measurements based on detection theory. IJST,
Transactions of Electrical Engineering, vol. 37, no. E2, pp. 101-120.

[27] Fan, L., Duan, H., and Long, F.2008 Face recognition by subspace analysis of 2D Log-Gabor wavelets features. 3rd International Conference on Intelligent System and Knowledge Engineering.

[28] Fischer, S., Sroubek, F., Perrinet, L., Redondo, R., and Cristóbal, G.2007 Self-invertible log-Gabor wavelets. International Journal of Computer, vol. 75, no. 2, pp. 231-246.

[29] Nava, R.2011 A comparison study of Gabor and logGabor wavelets for texture segmentation. 7th International Symposium on Image and Signal Processing and Analysis.

[30] Naperville Imaging Center $\mathrm{http} / / / \mathrm{www}$.napervillemri.com/mri-naperville-imagingcenter-chicago-il.html accessed on March 2015.

[31] Wang Aili., Gao Xue. and Gao Yue. (2014). A Modified Image Reconstruction Algorithm Based on Compressed Sensing, Fourth International Conference on Instrumentation and Measurement, Computer, Communication and Control. 